\title{
A Method for Detection of Repetitive Local Effects (RLEs) in Discrete Recursive Processes
}

\author{
E. KELMAN* and M. SHOSHANY \\ Department of Geography, Bar Ilan University, Ramat-Gan, 52900, Israel
}

(Received 8 September 1999)

\begin{abstract}
One-dimensional discrete processes, which can be measured repetitively for discrete values of arguments after discrete periodic or non periodic intervals of time, are discussed in this study. These processes can be under effect of different perturbations with different frequency, amplitudes, with accidental measurement error, etc. The problem investigated, is whether repetitive, local, relatively small effects (RLEs) can be extracted from this data. A simple method for such effects detection is proposed. A model for the simulation of discrete processes with local effects is introduced. Experiments with the method indicate that it is stable in detecting local effects and can be useful for many applications.
\end{abstract}

Keywords: Discrete time series; Filtering; Patterns recognition

\section{INTRODUCTION}

While most of the existing analysis of dynamic data (such as time series) is involved with separation between macro and meso-scale fluctuations, this study is interested at a special feature, some times at the level of noise. The characteristics of this phenomena is that it occurs persistently at certain locations in the sampling reference data domain (time, distance etc.), regardless to the magnitude of the macro and meso scale processes' amplitudes at these locations. This persistent appearance suggests that there is an important component of the dynamic processes and/or certain combination of local conditions forcing this phenomena. The mathematical form of these features has typical convexities or concavities, which appear with similar or different magnitudes in data series representing different samplings of the same dynamic processes. This phenomena will be termed here: Repetitive Local Effects (RLEs). Identification of RLEs is important since it may enable a better understanding of the factors determining the magnitude of the fluctuations at the different scales.

RLEs were first identified by Shoshany et al. (1999) in multi-temporal records of coastline changes. Examples for such phenomena are:

* Corresponding author. 
1. Small seismic features (Fig. 1) appearing in records acquired in two different places which might be related to the presence of certain tectonic structures or to an earthquake. 2. Temperature fluctuations (Fig. 2) that might occur following a certain pattern of regional synoptic conditions recorded in different cities around the world. 3. Absorption features (Fig. 3) at certain wavelength bands, occuring with different patterns of generalized reflectance distribution. 4. Influx of a spring to the streams' discharge as a (late) response to rainfall in certain parts of the drainage basin. 5. Effects of river depositions on seasonal shoreline fluctuations. 6. Local topographic effects on wind profiles.

The processes under investigation in this study are one-dimensional discrete processes measured several times for discrete values of arguments after discrete periodic or non periodic time intervals. These processes can be under effect of different perturbations with different frequency, amplitudes, etc. They can be measured with accidental errors. The problem that is investigated, is whether local, repetitive, relatively small effects (RLEs) can be extracted from this data. The word "local" in the above problem definition has two senses: 1 . Local effects connected with fixed values of argument that are significant only near these values. 2. Their amplitudes are relatively smallthey can be less than amplitudes of the accidental perturbations or errors.

Smoothing methods are not suitable for this problem because they can mask or remove effects with small amplitudes. In $[4,7]$ the filtering method introduced by Savitzky and Golay is applied for detecting the position of the absorption bands. Second, fourth and fifth order derivatives are numerically computed in the method. They are used in the simple criterion that is applied to each

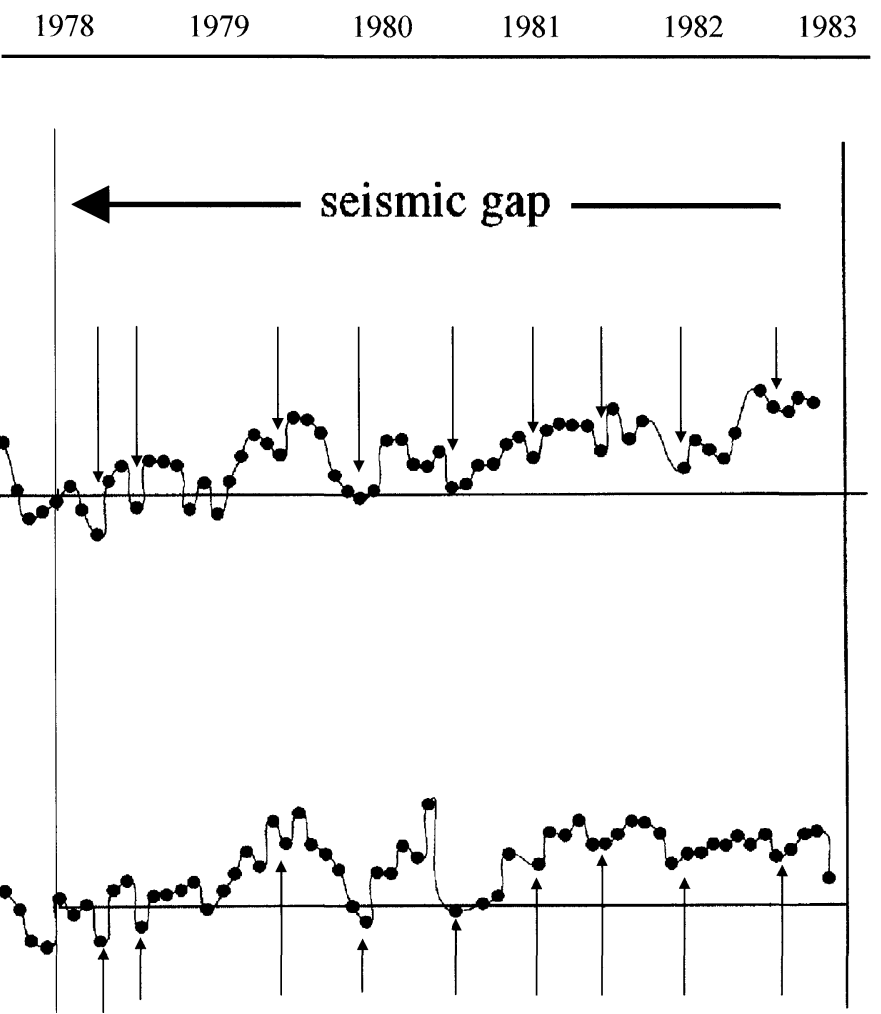

FIGURE 1 Records of seismic gaps in two different places (cf. Scholz, 1990). 


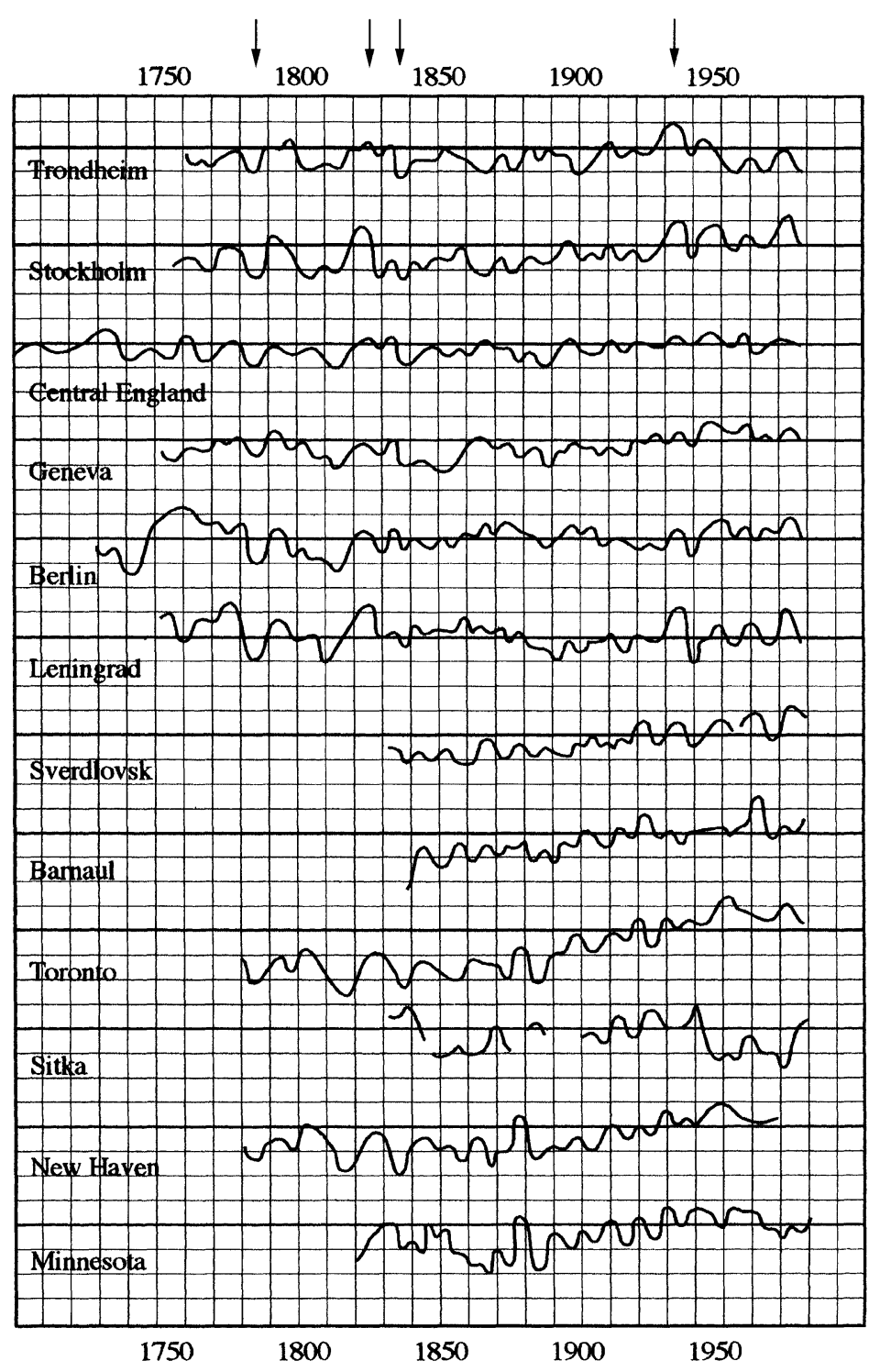

FIGURE 2 Temperature fluctuations ( $c f$. Bradley, 1995).

pixel of an image in order to detect an absorption band. Models of geophysical signals and noise, based on orthogonal functions of a discrete argument, are proposed in 3 .

Counts of the zero crossings from a time series after applying various families of filters may be used [3]. One of the major uses of higher order crossings is in the detection and estimation of discrete spectral components. In his book Morita (1995) emphasizes the applications and, in particular to pattern recognition. The book deals with the usual complex Fourier transform, the discrete Fourier transform, the fast Fourier transform and some others and their implementation.

These approaches have not applied yet to RLEs identification. Their application for the problem is difficult and possible effectiveness is not evident, since investigated data is not necessarily based on continuous functions and the noise can be of the scale of RLEs. Application of these techniques can 


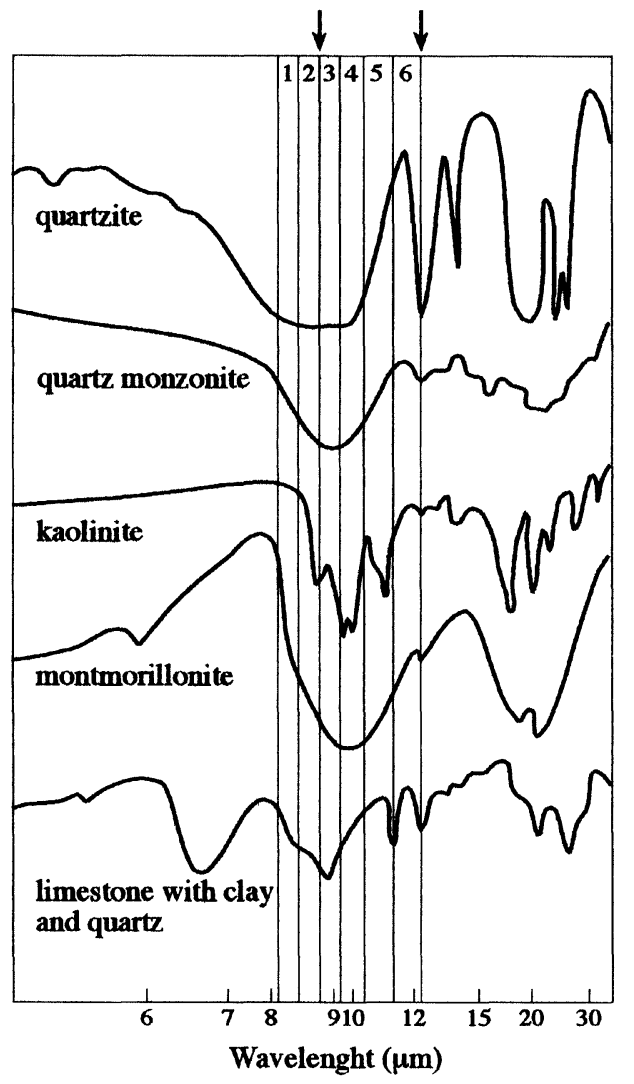

FIGURE 3 Absorption characteristics ( $c f$. Drury, 1993).

lead to artifacts, for example by increasing the order of approximating functions due to the above reasons.

The method, proposed in this study, is based on bend functions-functions that are introduced in the next section. They are simple to calculate and can be interpreted as a measure of the bending of discrete functions. When local extremal points of bend functions indicate positions of local effects is discussed. Numerical experiments with the method demonstrate that it is stable in detecting local effects, even when random errors and accidental perturbations are significant.

\section{THE METHOD}

Let us denote a discrete process as a function $p$ with discrete argument values $y_{i}$, and the number of argument values as $N$. Values $p\left(y_{i}\right)$ of this function will be denoted $p_{i}, i=1,2,3, \ldots, N$. It is supposed that these measurements are performed with a constant difference $h$ between the arguments:

$$
y_{i+1}-y_{i}=h, \quad i=1,2,3, \ldots, N-1 .
$$

The proposed method is as follows. A new function is defined:

$$
\begin{aligned}
z_{i} & =\sum_{j=1}^{k}\left(\frac{p_{i+j}+p_{i-j}}{2}-p_{i}\right), \\
i & =k+1, \ldots, N-k .
\end{aligned}
$$

Its points are analyzed in order to find extreme points. The number $k$ defines the number of terms in the sum of formula (1). In our study numbers from 5 to 15 were used for $k$ and all values $z_{i}$ were found with a fixed $k$. Consider now what extreme points of the function (1) indicate and how they can be used for our targets.

Let us initially suppose that function $p$ is a sufficiently smooth function of continuous argument. Then

$$
\begin{gathered}
\frac{p_{i+j}+p_{i-j}}{2}-p_{i}=\frac{\left(p_{i+j}-p_{i}\right)+\left(p_{i-j}-p_{i}\right)}{2}= \\
=\frac{1}{2}\left[\left(p^{\prime}\left(y_{i}\right) j h+\frac{1}{2} p^{\prime \prime}\left(y_{i}\right)(j h)^{2}\right)\right. \\
\quad+\left(-p^{\prime}\left(y_{i}\right) j h+\frac{1}{2} p^{\prime \prime}\left(y_{i}\right)(j h)^{2}\right) \\
\left.\quad+\tilde{O}_{j}\left[(j h)^{3}\right]\right]= \\
=\frac{1}{2} p^{\prime \prime}\left(y_{i}\right) j^{2} h^{2}+\tilde{O}_{j}\left[(j h)^{3}\right] .
\end{gathered}
$$

Therefore

$$
\begin{array}{r}
z_{i}=\frac{1}{2}\left(\sum_{j=1}^{k} j^{2}\right) p^{\prime \prime}\left(y_{i}\right) h^{2}+O_{k}\left[(h)^{3}\right]= \\
=A_{k} p^{\prime \prime}\left(y_{i}\right) h^{2}+O_{k}\left[(h)^{3}\right], \\
i=k+1, \ldots, N-k,
\end{array}
$$


where

$$
A_{k}=\frac{1}{2}\left(\sum_{j=1}^{k} j^{2}\right)
$$

and $A_{k}$ are factors that depend only on $k$ and do not depend on $i, p^{\prime \prime}\left(y_{i}\right)$ are second derivatives in points $y_{i}, \tilde{O}_{j}\left[(j h)^{3}\right]$ and $O_{k}\left[(h)^{3}\right]$ are infinitesimals of the third order. Therefore if $h$ is sufficiently small, the extreme points of function (1) correspond to extreme points of the second derivatives of function $p$. In other words, the bend of the function $p$ is maximal at these points.

This reasoning allows interpreting function (1), when $p$ is a discrete function. Function (1) can then be interpreted as a measure of bending of a discrete function. In this interpretation value $z_{i}$ is the measure of the bending of the discrete function $p$ at point $y_{i}$. We will call function (1) the $k$-bend function of the discrete process $p$. Use of several points in the sum of formula (1) allows decreasing the accidental error effect.

It is admissible to suppose that local effects are described by convex or concave functions. Bending of the function $p$ is then sharply changed in the local effect places. The above method therefore allows the indicating these places under certain conditions. An exact mathematical description of these conditions demands to find connections between variations of function $p$ and local effects, their smoothness, admissible amplitudes of random error, etc. However the other way is preferable: simulate typical situations and show the method opportunities on this basis. This is considered in two next sections.

\section{THE MODEL}

In this study discrete processes are simulated as a sum of three components: 1. Harmonic curves

$$
q_{i}(y)=a_{i} \sin \left(b_{i} y+f_{i}\right)
$$

with accidental parameters $a_{i}, b_{i}$ and $f_{i}$. 2. A local effects function. Local effects can be simulated by the function

$$
h(y)=g \exp \left(-d(c-y)^{2}\right) .
$$

The function has the form of a hump (see Fig. 4). Parameter $c$ defines its place, $g$ is equal to the maximal value of the function. Thus coordinates of the top of the "hump" are $(c, g)$. Parameter $d$ defines the width of the hump by means of the formula

$$
L=2 \frac{\sqrt{\ln 2}}{d}
$$

where $L$ denotes width of the hump at height $g / 2$.

3. Random error. This was simulated with the help of random number generator. Uniform probability distribution was used in a given segment. Periods $T_{i}$ of curves (3) and their phases $F_{i}$ at point $c$ are more convenient sometimes for

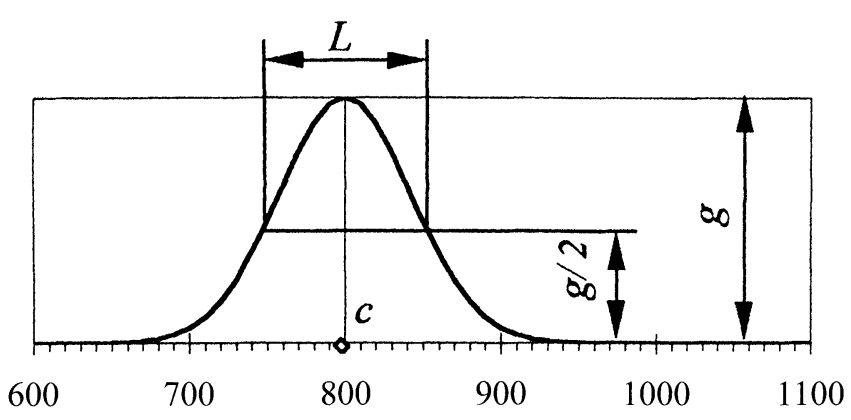

FIGURE 4 Local effects function. 
graph analysis and experiments instead of parameters $b_{i}$ and $f_{i}$. They can be calculated using formulas

$$
b_{i}=\frac{2 \pi}{T_{i}}, \quad f_{i}= \begin{cases}s_{i}, & \text { if } s_{i}<2 \pi, \\ s_{i}-2 \pi, & \text { if } s_{i} \geq 2 \pi,\end{cases}
$$

where

$$
s_{i}=2 \pi\left(1-\frac{c}{T_{i}}+\left[\frac{c}{T_{i}}\right]\right)+F_{i}
$$

and $[y]$ denotes the entire part of a real number $y$.

\section{EXPERIMENTS WITH THE MODEL}

A large number of numerical experiments with the model were performed. Different number of discrete processes was used for detection of local effect extraction. Different numbers of the harmonics (3) were used in these processes. The random choice of their parameters was done in different ranges. Different parameters of the local effect function (4) and random error amplitude were used. The ratio between parameter $g$ (height of the "hump"), on the one hand, and the amplitudes of

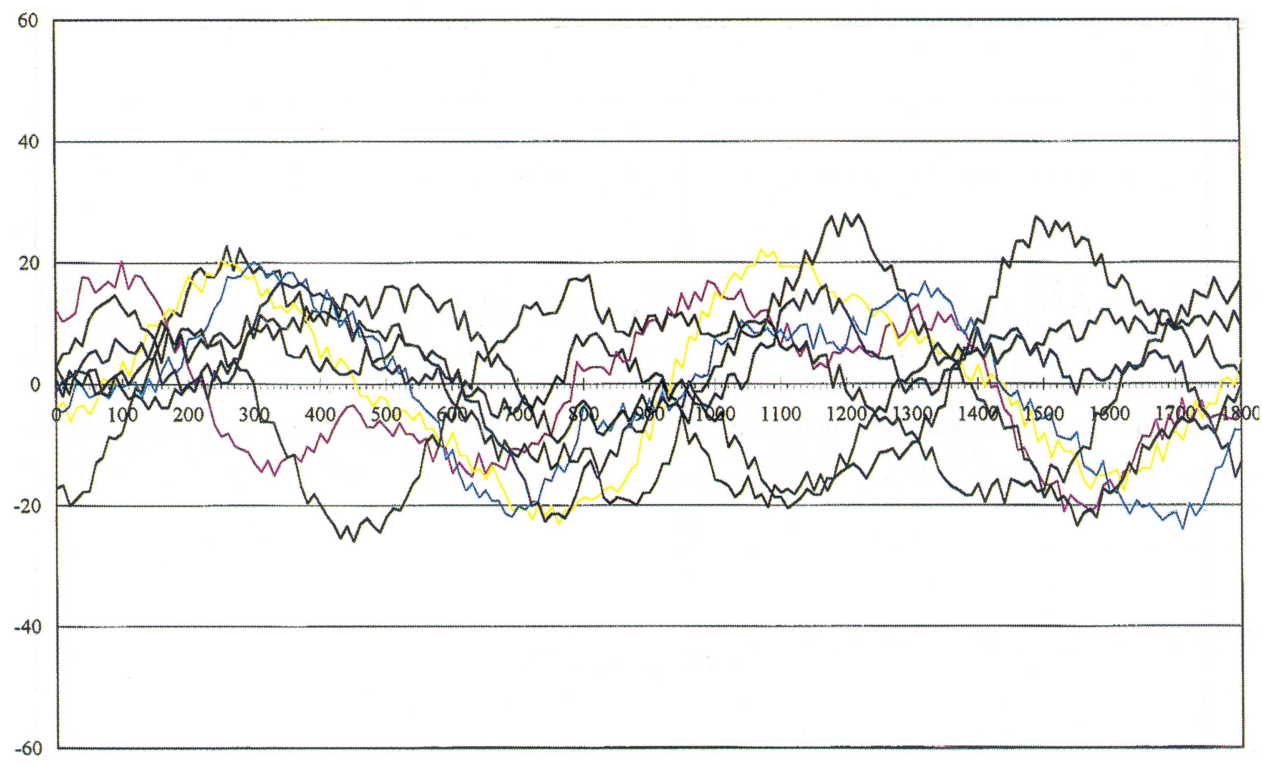

FIGURE 5 Graphs of the discrete processes (one can guess where is the RLE). (See Color Plate I.)

TABLE I Main parameters of the processes

\begin{tabular}{lcccccccc}
\hline Parameters & $\mathrm{A}$ & $\mathrm{B}$ & $\mathrm{C}$ & $\mathrm{D}$ & $\mathrm{E}$ & $\mathrm{F}$ & $\mathrm{G}$ & $\mathrm{H}$ \\
\hline$a_{1}$ & 4.9 & 4.6 & 3.1 & 3.8 & 2.7 & 4.6 & 2.7 & 3.9 \\
$a_{2}$ & 6.7 & 7.9 & 8.5 & 5.4 & 5.4 & 9.5 & 6.4 & 9.9 \\
$a_{3}$ & 10.6 & 14.6 & 15.1 & 12.0 & 15.5 & 12.6 & 12.6 & 14.1 \\
$T_{1}$ & 267.3 & 398.4 & 225.0 & 324.2 & 258.8 & 396.6 & 312.7 & 312.1 \\
$T_{2}$ & 584.5 & 464.1 & 598.6 & 438.9 & 505.0 & 717.7 & 412.9 & 633.4 \\
$T_{3}$ & 1046.0 & 977.4 & 923.6 & 1197.6 & 912.4 & 1068.7 & 1119.4 & 1152.8 \\
$F_{1}$ & 3.85 & 4.82 & 0.58 & 4.61 & 1.70 & 5.13 & 0.37 & 2.80 \\
$F_{2}$ & 4.35 & 0.70 & 5.70 & 3.57 & 2.91 & 4.20 & 3.52 & 2.42 \\
$F_{3}$ & 4.63 & 2.32 & 4.23 & 5.98 & 4.33 & 4.01 & 3.16 & 5.92 \\
$b_{1}$ & 0.024 & 0.016 & 0.028 & 0.019 & 0.024 & 0.016 & 0.020 & 0.020 \\
$b_{2}$ & 0.0108 & 0.0135 & 0.0105 & 0.0143 & 0.0124 & 0.0088 & 0.0152 & 0.0099 \\
$b_{3}$ & 0.0060 & 0.0064 & 0.0068 & 0.0052 & 0.0069 & 0.0059 & 0.0056 & 0.0055 \\
\hline
\end{tabular}


the harmonics and random error on the other hand, plays the most important role: the smaller this ratio, the less evident the local effect. Of course, experiments with different values of this ratio were performed.
The typical results of these experiments are presented in Figures 3-9. Figure 5 presents the graphs of 8 discrete processes. Parameters of the local effect are the same for all processes. The argument of the top of the local effect $c=800$,

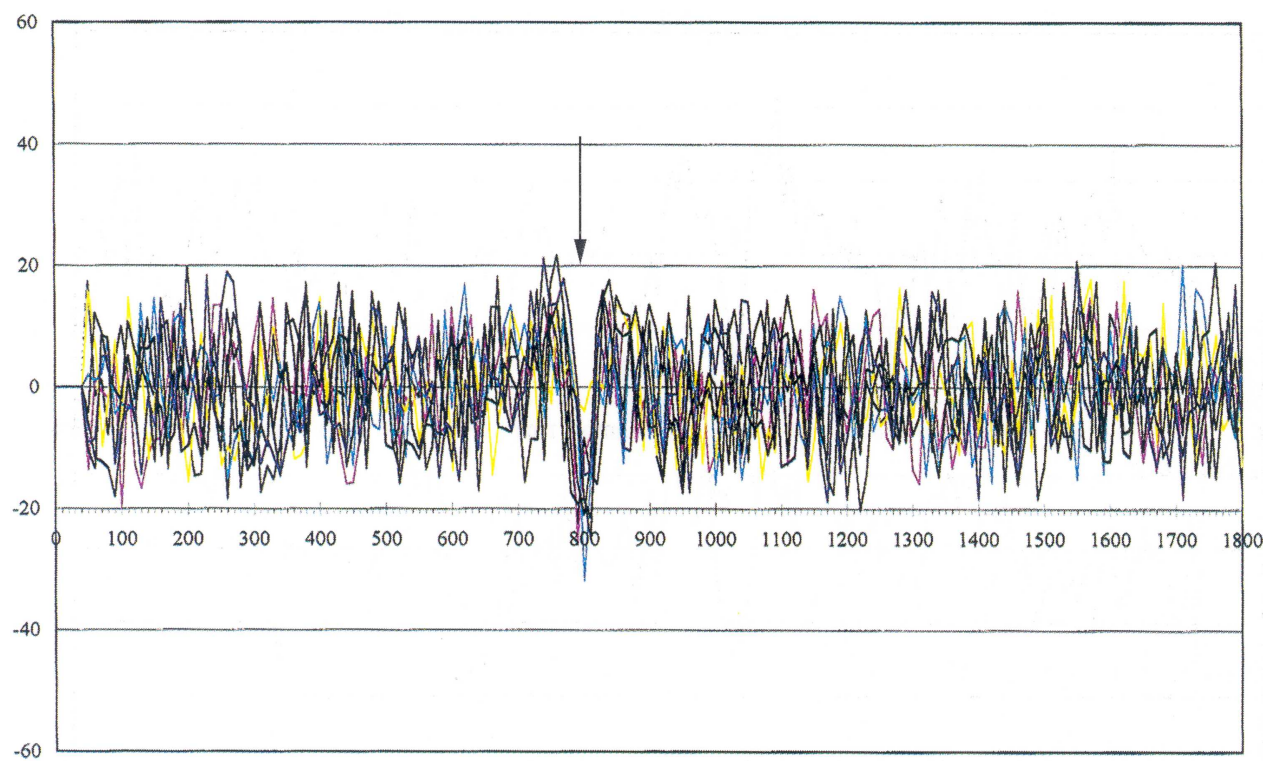

FIGURE 6 Detection of the RLE using of bend functions, $k=5$. (See Color Plate II.)

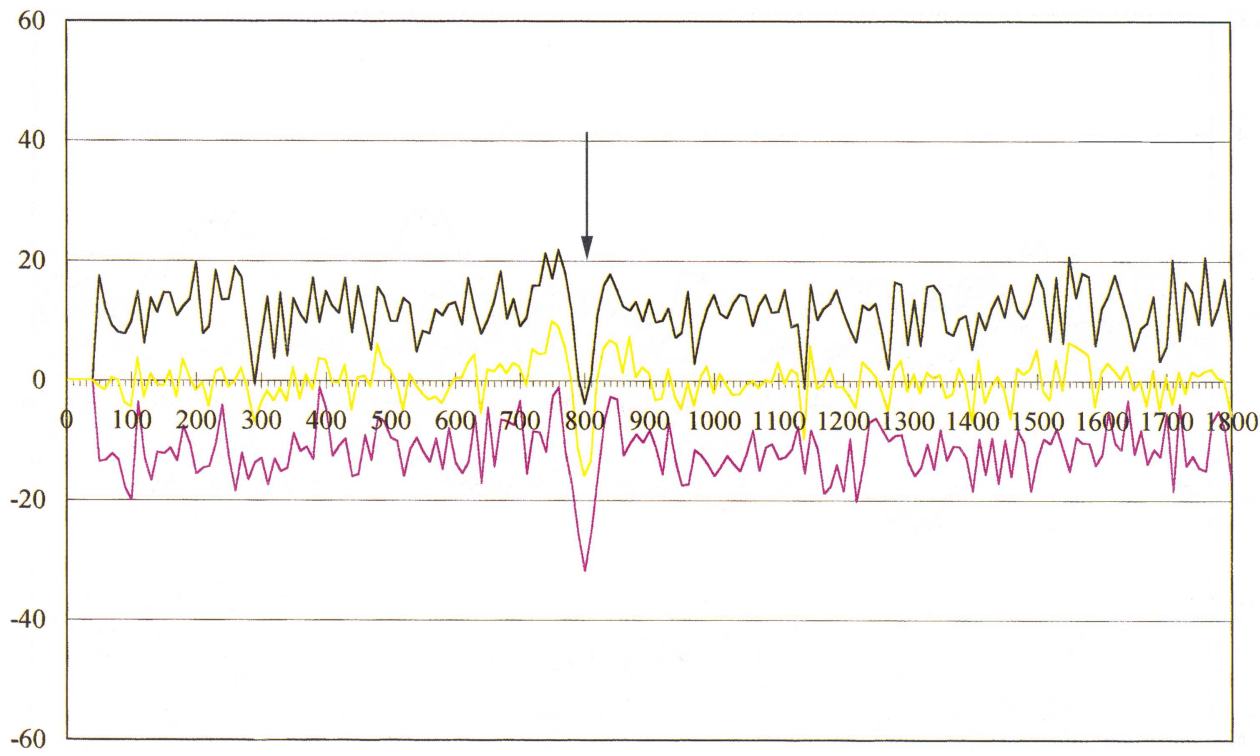

FIGURE 7 Detection of the RLE using of maximal, minimal and average functions, $k=5$. (See Color Plate III.) 


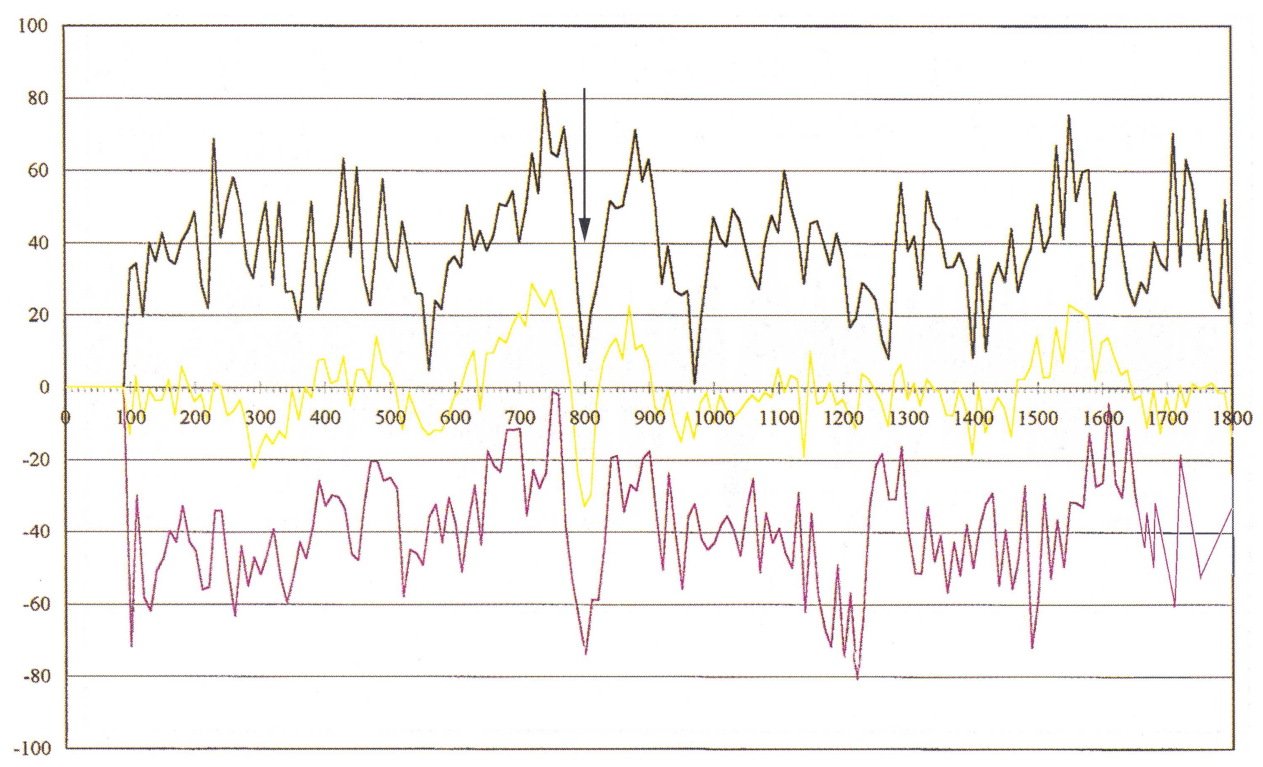

FIGURE 8 Detection of the RLE using of maximal, minimal and average functions, $k=10$. (See Color Plate IV.)

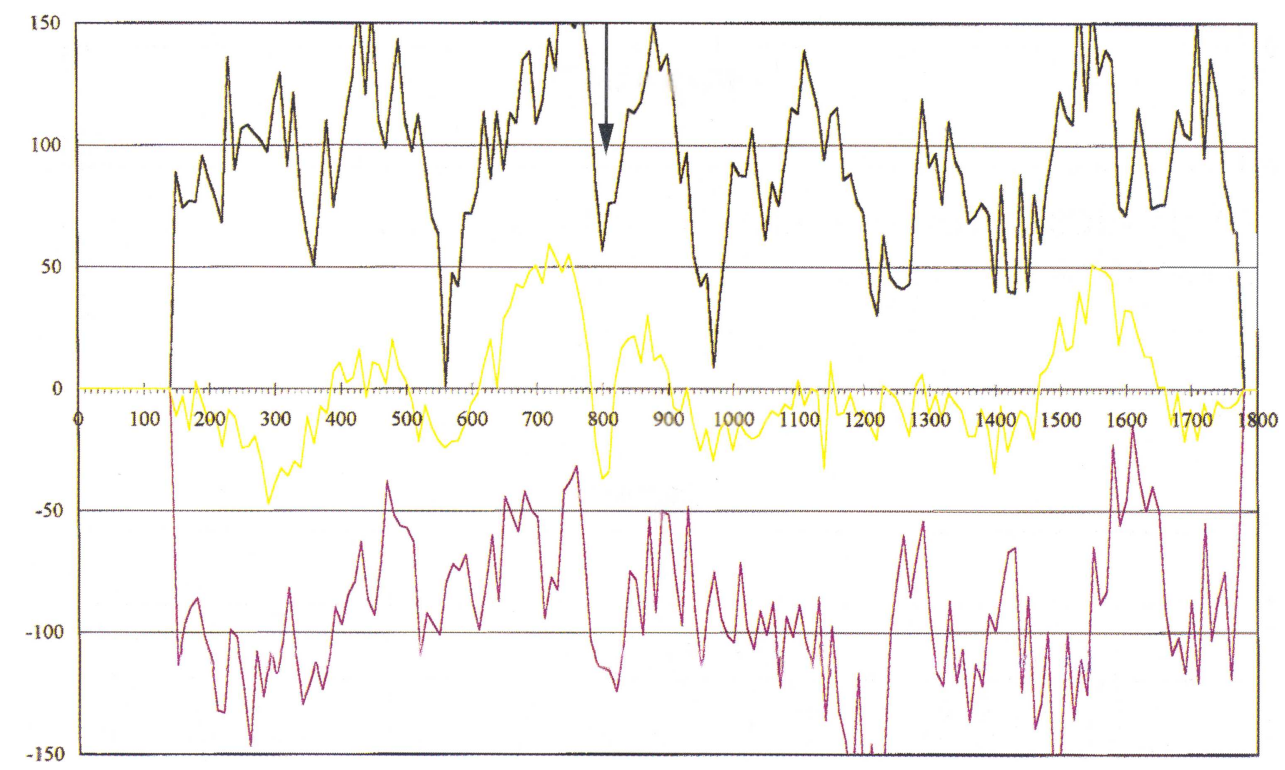

FIGURE 9 Detection of the RLE using of maximal, minimal and average functions, $k=15$. (See Color Plate V.)

its height $g=5, L=50$ and the local effect is not observed in the graphs of Figure 5.

The main parameters of these discrete processes are given in the Table I. Three harmonic curves with amplitudes $a_{i}$, periods $T_{i}$ and phases $F_{i}$ at point $c$ are included in each process. The amplitude of the random error was equal to 5 for each process. More exactly, random number within the range \pm 2.5 was added to every point of each process. 
Use of the proposed method is illustrated with the help of Figures 6-9. The formula (1) was used for calculating bend functions for each process with $k$ from 5 to 15 . A graph of the bend functions with $k=5$ is presented in Figure 6. The presence of the local effect at point $c=800$ is clearly indicated in the graphs.

Eight bend functions can be calculated for 8 discrete processes for each value of $k$. Maximal, minimal and average values of bend functions can be determined for every point of the argument. Graphs of these values are presented in Figures 7-9. These graphs are more convenient for analysis and detection of local effects. Also these graphs clearly indicate presence of the local effect: maximal, minimal and average functions have a joint local minimum at this point only.

\section{LOCAL EFFECTS DETECTION}

Unimodality of a function on a given interval is the property of having a unique extremum within this interval. The only interval where all functions - bend functions of all discrete processes with $k$ from 5 to 15 and their maximal, minimal and average functions-are uni-modal or almost unimodal in Figures 6-9 is the interval containing the local effect. Almost uni-modal means to be unimodal after an averaging with a small (1-3) number of adjacent points.

An interval containing a local effect can thus be defined using of the following properties:

(1) It is the interval where all functions (bend functions of all discrete processes with different $k$ and their maximal, minimal and average functions) are uni-modal or almost uni-modal.

(2) The length of the interval is significant.

(3) The above properties 1 and 2 hold for $k$ in a relatively wide range.

Experience based on our experiments indicates that the recommended minimal length of the interval of the property 2 and range of the property 3 should be five. The following procedure can be used for RLEs detection:

0 . Define initial and final values for $k$.

1. Find the bend functions, their maximal, minimal and average functions for all $k$.

2. Find local extrema of these functions. Find intervals where they are uni-modal or almost uni-modal.

3. Select intervals where all functions are unimodal or almost uni-modal.

\section{CONCLUSIONS}

Bend functions of a discrete one-dimensional process were introduced in this study. The method based on bend functions was proposed for detecting local effects in these processes. A model for the simulation of discrete processes with local effects was used for experiments using this method. Experiments with the method indicate that it is stable in detecting local effects, even when random error is equal to the amplitude of the local effect and when it is significantly less than the amplitudes of perturbations. This method may be useful for detection repetitive local effects in spectral plots, electrocardiograms, many processes in hydrography and meteorology, etc. Its application for real processes have begun and have afforded interesting results.

\section{References}

[1] Bradley, R. S. and Jones, P. D. (1995). Climate since A.D. 1500 , Routledge, London and New York, 706 pp.

[2] Drury, S. A. (1993). Image interpretation in Geology, Chapman \& Hall, London, $\mathrm{xi}+283$.

[3] Kedem, B. (1994). Time series analysis by higher order crossings. IEEE Press, New York, xiv + 318 pp.

[4] King, R. L., Ruffin, C., LaMastus, F. E. and Shaw, D. R. (1999). The analysis of hyperspectral data using SavitzkyGolay filtering - Practical Issues, IGARSS'99 IEEE 1999 International Geoscience and Remote Sensing Simposium, Hamburg, CD ROM-IEEE Catalog Number 99CH36293.

[5] Marchenko, V. B. (1992). Orthogonal functions of a discrete argument and their application in geophysics, Naukova Dumka, Kiev, 211 pp. 
[6] Morita, K. (1995). Applied Fourier transform. IOS Press, Amsterdam, xii $+433 \mathrm{pp}$.

[7] Rufin, C. and King, R. L. (1999). The analysis of hyperspectral data using Savitzky-Golay filteringTheoretical basis, IGARSS'99 IEEE 1999 International Geoscience and Remote Sensing Simposium, Hamburg, CD ROM - IEEE Catalog Number 99CH36293.
[8] Scholz, C. H. (1990). The mechanics of earthquakes and faulting, Cambridge University Press, New York, 439 pp.

[9] Shoshany, M., Golik, A., Kelman, E. and Galili, E. (1999). The detection of coastal morphological features from multi date aerial photography: the case study of Haifa's shoreline, Israel, Photog. Eng. and Remote Sensing (in preparation). 


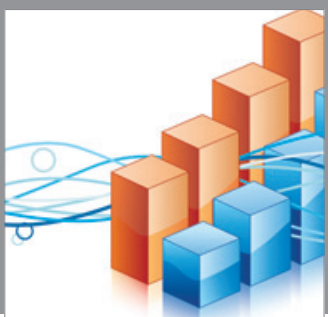

Advances in

Operations Research

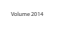

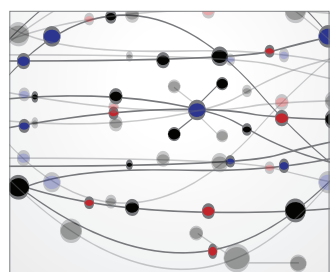

\section{The Scientific} World Journal
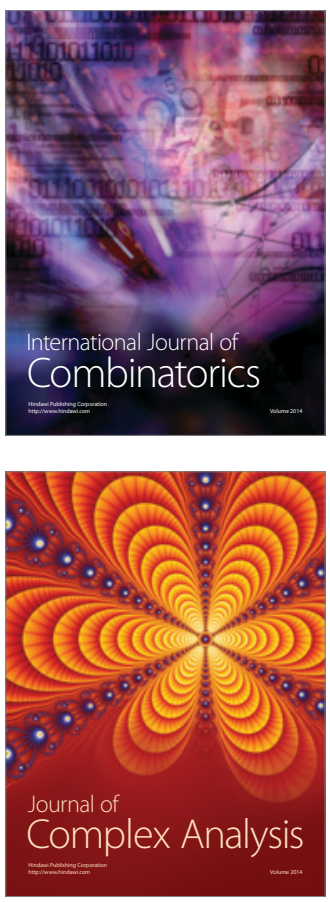

International Journal of

Mathematics and

Mathematical

Sciences
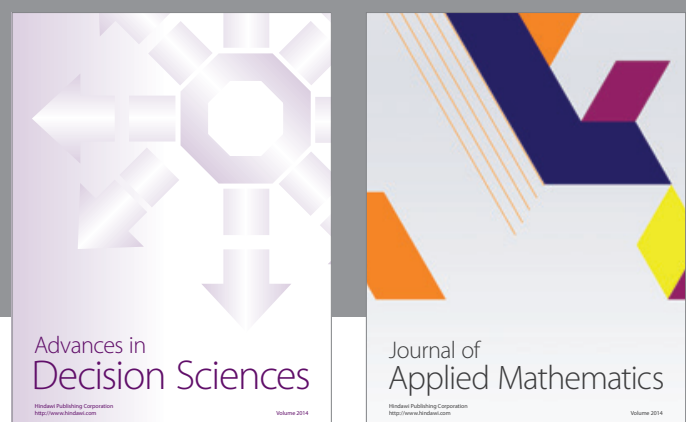

Journal of

Applied Mathematics
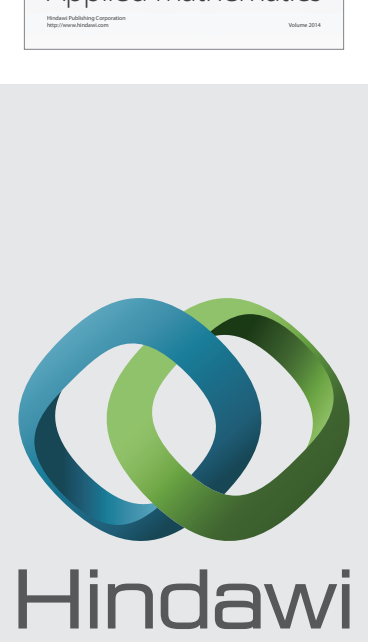

Submit your manuscripts at http://www.hindawi.com
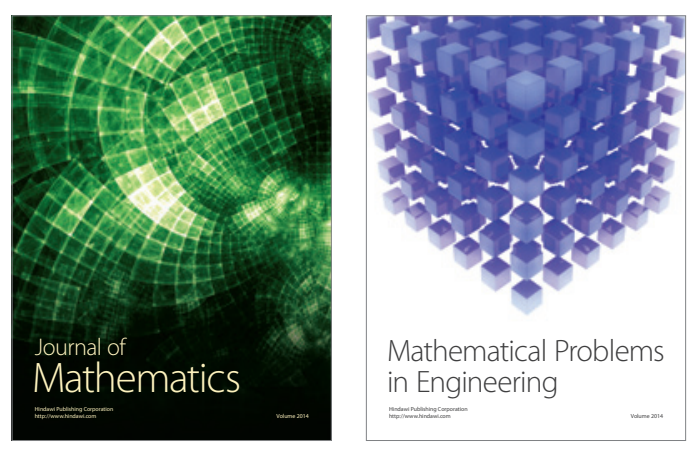

Mathematical Problems in Engineering
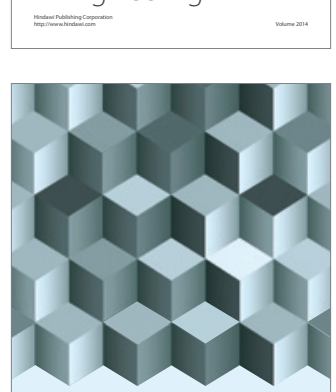

Journal of

Function Spaces
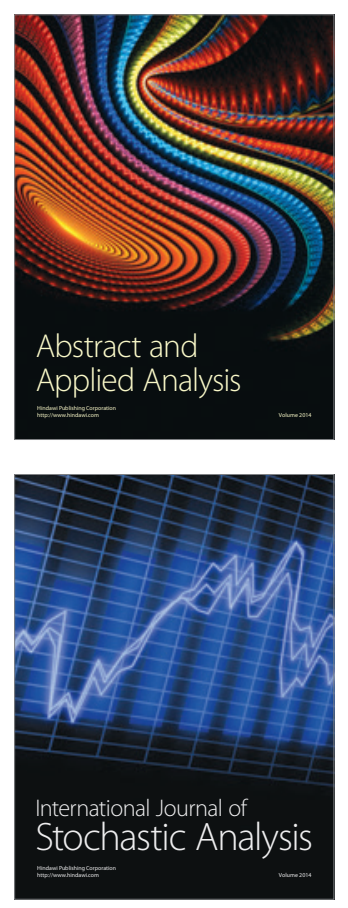

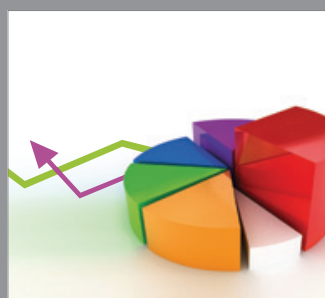

ournal of

Probability and Statistics

Promensencen
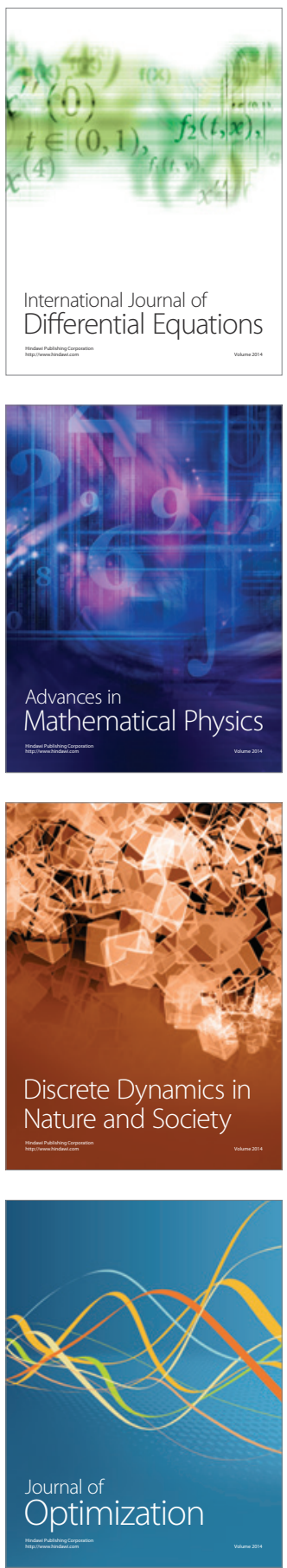